\title{
OPTIMIZATION OF COMPLEX SYSTEMS IN THE PRESENCE OF UNCERTAINTY
}

Ralph Rockafellar

UNIVERSITY OF WASHINGTON

09/08/2014

Final Report

DISTRIBUTION A: Distribution approved for public release.

Air Force Research Laboratory

AF Office Of Scientific Research (AFOSR)/ RTA

Arlington, Virginia 22203

Air Force Materiel Command 


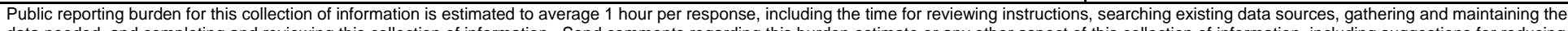

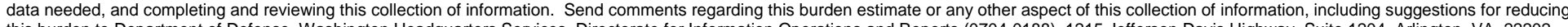

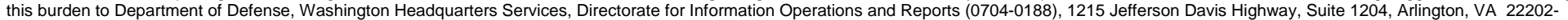

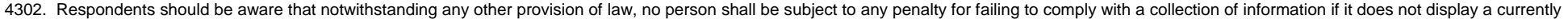
valid OMB control number. PLEASE DO NOT RETURN YOUR FORM TO THE ABOVE ADDRESS.
1. REPORT DATE (DD-MM-YYYY)
2. REPORT TYPE
3. DATES COVERED (From - TO)

08-24-2014

FINAL

4. TITLE AND SUBTITLE

Optimization of Complex Systems in the Presence of Uncertainty and Approximations

$08 / 01 / 2001-07 / 31 / 2014$

5a. CONTRACT NUMBER

5b. GRANT NUMBER

FA9550-11-1-0206 MOD P0

5c. PROGRAM ELEMENT NUMBER

6. AUTHOR(S)

Ralph Tyrrell Rockafellar 5d. PROJECT NUMBER

5e. TASK NUMBER

5f. WORK UNIT NUMBER

8. PERFORMING ORGANIZATION REPORT NUMBER

\section{University of Washington}

7. PERFORMING ORGANIZATION NAME(S) AND ADDRESS(ES)

3917 University Way NE

Seattle, WA 98105-6692

9. SPONSORING I MONITORING AGENCY NAME(S) AND ADDRESS(ES)

10. SPONSOR/MONITOR'S ACRONYM(S)

Air Force Office of Science and Research

875 Randolph Street

Suite 325 Room 3112

Arlington, VA 22201

11. SPONSOR/MONITOR'S REPORT NUMBER(S)

\section{DISTRIBUTION / AVAILABILITY STATEMENT}

Distribution A - Approved for public release

\section{SUPPLEMENTARY NOTES}

\section{ABSTRACT}

Engineering decisions are invariably made under substantial uncertainty about current and future system cost and response, including cost and response associated with low-probability but high-consequence events. Such events motivate approaches that typically have centered on constraining or minimizing probability of failure, in contrast to the risk-neutral approach of constraining or minimizing expected values. The research under this proposal has, instead, developed concepts of risk-averse decision making between these extremes with the aim of achieving an advanced methodology better able to deal with risks and reliability in engineering design.

Measures of risk that go beyond statistical quantiles to so-called superquantiles (CVaR) and their mixtures have been the main focus. The results have explored their superior properties and enhanced computability along with surprising implications that standard least-squares regression in statistical approximations might better be supplanted by generalizations like quantile and even superquantile regression. Superquantile regression, which provides a cautious and powerful tool, is completely new. It is entirely a product of this grant research.

\section{SUBJECT TERMS}

engineering risk, reliable design, optimization, regression, probability of failure, measures of risk, quantiles, superquantiles

\begin{tabular}{|c|c|c|c|c|c|}
\hline \multicolumn{3}{|c|}{ 16. SECURITY CLASSIFICATION OF: } & \multirow{2}{*}{$\begin{array}{l}\text { 17. LIMITATION } \\
\text { OF ABSTRACT } \\
\text { UU }\end{array}$} & \multirow{2}{*}{$\begin{array}{l}\text { 18. NUMBER } \\
\text { OF PAGES } \\
5\end{array}$} & $\begin{array}{l}\text { 19a. NAME OF RESPONSIBLE PERSON } \\
\text { Ralph Tyrrell Rockafellar }\end{array}$ \\
\hline $\begin{array}{l}\text { a. REPORT } \\
\text { UU }\end{array}$ & $\begin{array}{l}\text { b. ABSTRACT } \\
\text { UU }\end{array}$ & $\begin{array}{l}\text { c. THIS PAGE } \\
\text { UU }\end{array}$ & & & $\begin{array}{l}\text { 19b. TELEPHONE NUMBER (include area } \\
\text { code) } \\
206-849-3249\end{array}$ \\
\hline
\end{tabular}


Final Report for "Optimization of Complex Systems in the Presence of Uncertainty and Approximations"

\section{Summary of Effort}

The research was a collaborative effort with Johannes Royset of the Naval Postgraduate School, who had separate funding from AFOSR. The PI received one month of salary in each of the three years for his research, which closely followed the lines of the proposal.

\section{Accomplishments and New Findings}

Engineering decisions are invariably made under substantial uncertainty about current and future system cost and response, including cost and response associated with low-probability, high-consequence events. A risk-neutral decision maker would rely on expected values when comparing designs, while a risk-averse decision maker might adopt nonlinear utility functions or failure probability criteria. Paper [2] shows that these models for making decisions fall within a framework of risk measures that includes many other possibilities. General recommendations for selecting risk measures lead to decision models for risk-averse decision making that comprehensively represent risks in engineering systems, avoid paradoxes, and accrue substantial benefits in subsequent risk, reliability, and cost optimization. The paper provides an overview of the framework of decision making based on risk measures.

Superquantile risk, also known as conditional value-at-risk (CVaR), is widely used as a coherent measure of risk due to its improved properties over those of quantile risk (valueat-risk). The latest paper [1] considers second-order superquantile/CVaR measures of risk, which represent further "smoothing" by averaging the classical quantities. It also steps further and examines the more general "mixed" superquantile/CVaR measures of risk with fundamental importance in dual utility theory. It establishes representations of these mixed and second-order superquantile risk measures in terms of risk profiles, risk envelopes, and risk identifiers. The expressions facilitate the development of dual methods for mixed and 
second-order superquantile risk minimization as well as superquantile regression, a secondorder version of quantile regression.

Superquantiles (also called conditional values-at-risk) are useful tools in risk modeling and optimization, with expanding roles beyond these areas. Paper [5] provides a broad overview of superquantiles and their versatile applications. We see that superquantiles are as fundamental to the description of a random variable as the cumulative distribution function (cdf), they can recover the corresponding quantile function through differentiation, they are dual in some sense to superexpectations, which are convex functions uniquely defining the cdf, and they also characterize convergence in distribution. A superdistribution function defined by superquantiles leads to higher-order superquantiles as well as new measures of risk and error, with important applications in risk modeling and generalized regression.

Random variables can be described by their cumulative distribution functions, a class of nondecreasing functions on the real line. Those functions can in turn be identified, after the possible vertical gaps in their graphs are filled in, with maximal monotone relations, and this is the theme of paper [3], which provides a new foundation for superquantile analysis. Maximal monotone relations are known to be the subdifferentials of convex functions. Analysis of these connections yields fresh insights. The generalized inversion operation between distribution functions and quantile functions corresponds to graphical inversion of monotone relations. In subdifferential terms, it corresponds to passing to conjugate convex functions under the Legendre-Fenchel transform. Among other things, this shows that convergence in distribution for sequences of random variables is equivalent to graphical convergence of the monotone relations and epigraphical convergence of the associated convex functions. Measures of risk that employ quantiles ( VaR) and superquantiles (CVaR), either individually or in mixtures, are illuminated in this way. Formulas for their calculation are seen from a perspective that reveals how they were discovered. The approach leads further to developments in which the superquantiles for a given distribution are interpreted as the quantiles for an overlying "superdistribution." In this way a generalization of Koenker-Basset error is derived which provides a platform for superquantile regression as a higher-order extension 
of quantile regression.

Paper [4] presents a generalized regression technique centered on a superquantile (also called conditional value-at-risk) that is consistent with that coherent measure of risk and yields more conservatively fitted curves than classical least-squares and quantile regression. In contrast to other generalized regression techniques that approximate conditional superquantiles by various combinations of conditional quantiles, we directly and in perfect analog to classical regression obtain superquantile regression functions as optimal solutions of certain error minimization problems. We show the existence and possible uniqueness of regression functions, discuss the stability of regression functions under perturbations and approximation of the underlying data, and propose an extension of the coefficient of determination R-squared for assessing the goodness of fit. The paper presents two numerical methods for solving the error minimization problems and illustrates the methodology in several numerical examples in the areas of uncertainty quantification, reliability engineering, and financial risk management.

\section{Personnel Supported}

Personnel supported under the grant: Prof. R.T. Rockafellar, University of Washington, Seattle. Students involved but not supported: S. Miranda (Naval Postgraduate School).

\section{Publications}

The following publications were written under the project;

see http://www.math.washington.edu/ rtr/mypage.html for access to the papers.

1. R.T. Rockafellar and J.O. Royset, "Superquantile/CVaR Risk Measures: Second-Order Theory," in review.

2. R.T. Rockafellar and J.O. Royset, "Engineering Decisions under Risk-Averseness," in review. 
3. R.T. Rockafellar and J.O. Royset, 2014, "Random Variables, Monotone Relations, and Convex Analysis," Mathematical Programming B, to appear.

4. R.T. Rockafellar, J.O. Royset, and S.I. Miranda, 2014, "Superquantile Regression with Applications to Buffered Reliability, Uncertainty Quantification, and Conditional Value-at-Risk," European Journal of Operational Research, Vol. 234, No. 1, pp. 140154.

5. R.T. Rockafellar and J.O. Royset, 2013, "Superquantiles and Their Applications to Risk, Random Variables, and Regression," INFORMS Tutorials, INFORMS.

\section{Interactions/Transitions}

The project resulted in the following presentations by R.T. Rockafellar:

1. Feb 2012: "Uncertainty, Risk and Statistics," Workshop on Information Technology, University of Florida, Gainesville, FL.

2. Sep 2012: "Random Variables, Monotone Relations and Convex Analysis," Conference on Nonlinear Optimization and Applictions, Matsue, Japan.

3. Sep 2012: "Random Variables, Monotone Relations and Convex Analysis," Optimization seminar, University of Paris VI, France.

4. Sep 2012: "The Modeling of Risk in Optimization Under Uncertainty," PGMO Conference, Ecole Polytechnique, Paris, France.

5. Oct 2012: "Random Variables, Monotone Relations and Convex Analysis," Workshop on Optimization, Polytechnical University, Hong Kong.

6. Oct 2012: "Random Variables, Monotone Relations and Convex Analysis," Optimization Seminar, University of Chile, Santiago.

7. Dec 2012: "The Modeling of Risk in Optimization Under Uncertainty," 5th OCA Conference, Beijing, China. 
8. Feb 2013: "Random Variables, Monotone Relations and Convex Analysis," iSouth Pacific Optimization Meeting, University of Newcastle, Australia.

9. May 2013: "Risk, Optimization and Statistics," University of Goettingen, Germany.

10. Jun 2013: "The Fundamental Quadrangle of Risk in Optimization and Statistics," International Conference on Optimization and Statistics, Beijing, China.

11. May 2014: "The Fundamental Quadrangle of Risk in Optimization and Statistics," SIAM Conference on Optimization, San Diego, California.

12. Jun 2014: "The Fundamental Quadrangle of Risk in Optimization and Statistics," Optimization seminar, University of Groningen, Netherlands.

\section{New discoveries, inventions, or patent disclosures}

No patents, but several discoveries and advances as described above. 